Gastrointestinal disease

Anorexia

Constipation

Colonic stricture

Crohn's disease

Diverticulosis

Duodenal abnormality

Esophageal varices

Esophagitis

Fecal impaction

Functional bowel disease

Gastric ulcer

Gastritis

Gastroenteritis

Gastrointestinal bleeding

Ischemic colitis

Malabsorption

Mallory Weiss tear

Nausea

Pancreatitis

Peptic ulcer

Pyloric stenosis

Volvulus

Unspecified gastrointestinal abnormality

Pulmonary disease

Asthma

Bronchiectasis

Bronchiolitis
Bronchitis

Chronic lung disease

Coin lesion

Empyema

Interstitial fibrosis

Lobar atelectasis

Mediastinal mass

Pleural effusion

Pneumoconiosis

Pneumonia

Pneumothorax

Pulmonary cancer

Pulmonary embolism

Pulmonary mass

Active tuberculosis

Unspecified respiratory abnormality

Renal disease

Glomerulonephritis

Renal hypertension

Hepatorenal syndrome

Hydronephrosis

Polycystic kidney disease

Diabetic nephropathy

Nephrotic syndrome

Renal insufficiency

Unspecified renal abnormality

Cancer of any origin

\title{
REFLECTIONS
}

\section{At General Hospital}

We walk into Miss Randall's room

Just when the TV doctors do.

Us through the door, them through the screen;

We're Med Team One, they're Channel Two.

We make rounds as they make rounds

All rounding the bed.

I think: what thoughts of us and them

Are rounding in her head?

The TV docs she's never met,

but through the tube she's seen

Their hopes and fears and hidden lusts.

It's all there on the screen.

And though we see her every day,

We talk and touch her force to face.

Compared with them we must seem dull -

We have no lives outside this place.
Or does she build us secret lives With TV hopes and TV fears,

Filled with heartbreak and intrigue,

Complete with television tears?

Whom has she picked to be the star? Whose faltering career is through? Which of us stabs the others' backs? Which of us goes to bed with who?

I wonder if she watches them

To see that secret world in me.

Or might she place her trust in us

Because we look just like TV?

Greg Simon, $M D$

12 Carver Street

Cambridge, Massachusetts 